\title{
Unorthodox routes to prostanoid formation: new twists in cyclooxygenase-initiated pathways
}

\author{
Charles N. Serhan ${ }^{1}$ and Ernst Oliw ${ }^{2}$ \\ ${ }^{1}$ Center for Experimental Therapeutics and Reperfusion Injury, Department of Anesthesiology, Perioperative and Pain Medicine, \\ Brigham and Women's Hospital and Harvard Medical School, Boston, Massachusetts, USA \\ ${ }^{2}$ Division of Biochemical Pharmacology, Department of Pharmaceutical Biosciences, \\ Uppsala Biomedical Center, Uppsala University, Uppsala, Sweden \\ Address correspondence to: Charles N. Serhan, Center for Experimental Therapeutics and Reperfusion Injury, \\ Department of Anesthesiology, Perioperative and Pain Medicine, Brigham and Women's Hospital and Harvard Medical School, \\ Boston, Massachusetts 02115, USA. Phone: (617) 732-8822; Fax: (617) 278-6957; E-mail: cnserhan@zeus.bwh.harvard.edu.
}

Since their discovery in the mid 1930s as potent bioactive compounds in human semen (1), prostaglandins and their relatives have fascinated several generations of scientists. Prostanoids, as they are known today, are a specific class of bioactive mediators generated via initial actions of cyclooxygenase. They are part of a much larger group of compounds, the eicosanoids, which, although ubiquitous as a group, are produced by a variety of cell type-, tissue-, and species-specific biosynthetic pathways. The eicosanoid family encompasses thromboxanes, prostacyclins, leukotrienes, hydroxyeicosatetraenoic acids (HETEs), epoxyeicosatrienic acids (EETs), lipoxins, and isoprostanes, the last of which can be generated by nonenzymatic oxidative mechanisms and may serve as markers of oxidative stress in humans. Each class of enzymatically produced eicosanoids governs a specific set of functions and is thought to serve as a mediator or autacoid, acting within a local microenvironment to orchestrate key cellular responses. A goal of this Perspective is to provide a succinct view of salient findings and historical background in this area necessary for the reader to evaluate the potential importance of the newly uncovered "unorthodox" routes (2-4) initiated via cyclooxygenase (for detailed reviews see refs. 5-7).

Structures of the first six prostaglandins (PGs) were established by 1962, before their individual biosynthetic pathways were studied. These compounds are synthesized in vivo through what can now be regarded as the "orthodox" cyclooxygenase pathways, which came to light largely through the work of Sune Bergström, who led a team then based at the Karolinska Institutet in Stockholm. Bergström stated in his 1982 Nobel lecture that, following the structural assignments of the six PGs, "These 20carbon prostaglandins have cis-double bonds located as in certain essential fatty acids, when counting from the carboxyl. This made us suspect that these naturally occurring acids might be precursors" (see ref. 5 and references within). Indeed, evidence obtained with isotopically labeled 20-carbon fatty acids incubated with sheep vesicular glands, an abundant source of PGs, confirmed this assumption. More importantly, this finding shattered a dogma of the time that held that fatty acids played only structural roles in cells, thus opening the way for discovery of the many other important bioactive fatty acid-derived products. These compounds fall into several distinct classes and include the leukotrienes (LTs) and the lipoxins (lipoxygenase interaction products or LXs; see ref. 9). Eicosanoids play pivotal roles in parturition, inflammation, hemodynamics, and renal function, and our appreciation of their bioimpact and relevance to human diseases is still evolving. Here, we focus on newly uncovered pathways, involving either the cyclooxygenases (COXs) or nonenzymatic chemical transformations, that lead to the formation of bioactive prostanoids and of previously unknown lipid mediators produced by COX-2.

Cyclooxygenase, biosynthesis of prostaglandin $\mathrm{H}$, and the impact of aspirin

The tissue-specific biosynthetic pathways that generate specific PGs are well covered in textbooks. In these reactions, COX carries out a complex free radical reaction by acting successively as a bis-dioxygenase and a peroxidase (Figure 1, left). This dual role was first postulated over 35 years ago $(6,8)$ but can now be rationalized by models from crystal structures of the enzyme $(9,10)$.

The orthodox pathway, as it entered the literature in the 1960s, is as follows (Figure, 1, left): Receptor activation or cell injury signals for arachidonic acid release from membrane stores. The activity of lipases such as cytosolic phospholipase $\mathrm{A}_{2}$ (reviewed in Fitzpatrick and Soberman, this Perspective series, ref. 11) is typically the limiting factor at this step. COX catalyzes the cyclization of arachidonic acid to $\mathrm{PGH}_{2}$ - hence its other name, prostaglandin $\mathrm{H}$ synthase or PGHS $(5,6)$. COX accomplishes this by first generating $\mathrm{PGG}_{2}$, after which the peroxidase part of the enzyme acts, reducing the carbon 15 position hydroperoxide to its corresponding alcohol to form $\mathrm{PGH}_{2}$ (Figure 1). These reactions are initiated by stereoselective abstraction of hydrogen at carbon number 13 (in the $S$ configuration), which guides the molecule oxygen insertion by COX to form the characteristic endoperoxide bridge between carbon 9 and 11 and the 
five carbon member ring (see $\mathrm{PGG}_{2}$; Figure 1) that defines the PGs. The bis-dioxygenase activity (insertion of a second molecule of $\mathrm{O}_{2}$ ) involves a radical center formed at carbon 15 to yield a hydroperoxy group in $\mathrm{PGG}_{2}$, which is reduced to the alcohol $\mathrm{PGH}_{2}(12)$. The next step, namely conversion of $\mathrm{PGH}_{2}$ to cell type-specific production of individual prostanoids, is governed by enzymes that are expressed in both a tissue- and cell type-selective fashion. Thus, for example, $\mathrm{PGH}_{2}$ gives rise to prostacyclin in endothelial cells and to thromboxane in platelets (6).

The preferred substrates of COX contain at least three double bonds in well-defined positions. These are dihomogammalinolenic, arachidonic, and eicosapentaenoic (EPA) acids, which contain 3, 4, and 5 double bonds, respectively. Other fatty acids lacking the three double bonds in the required positions can be oxygenated by COX to hydroperoxy fatty acids, but not to PGs. Thus, Mead acid (C20:3n-9) is transformed to 13hydroxy-5,8,11-eicosatrienoic acid by COX-1 (13), and there are many exciting new transformations of different new substrates of COX enzymes, and particularly of COX-2 $(3,14,15)$, as discussed below.

The relevance of aspirin (acetylsalicylic acid; ASA), the founding member of the nonsteroid anti-inflammatory drugs (NSAIDs), to the events in this classical pathway was established by John Vane and colleagues in the 1970s. However, ASA had been in wide use as an analgesic, antipyretic, and anti-inflammatory drug since 1899, despite the absence of a molecular mechanism for its actions. By 1970, several new NSAIDs had been added to the medicine cabinet, and their mechanisms were likewise unknown. Vane's group discovered that ASA inhibits PG formation and developed the hypothesis that this biochemical intervention by NSAIDs is the basis of their therapeutic actions and side effects (7) (Figure 1, left).

The unanticipated arrival of COX-2

COX-1, first purified in 1976, was cloned in 1988. At the time, $P G$ generation was assumed to involve only the one COX enzyme, but in 1991 several groups independently reported a second gene with COX activity, an isoform denoted COX-2 (ref. 16; see also Smith and Langenbach, this Perspective series, ref. 17). The COX-2 isoform is expressed in many cell types in vitro and during inflammation in vivo, suggesting that, whereas COX-1 generates a constitutive pool of prostanoids that can maintain cellular homeostasis, the induction of COX-2 might explain the increased levels of prostanoids that accompany various pathologies. COX-1 and COX-2 are products of different genes, and although their amino acid identity is only about $61 \%$ overall (18), their three-dimensional $x$-ray crystal structures are virtually superimposable. Both enzymes catalyze the same reactions (Figure 1), making selective inhibition of one isoform over the other a formidable challenge. Several pharmaceutical companies have developed COX-2 inhibitors with the hope that new chemical classes of inhibitors display the analgesic and anti-inflammatory properties of traditional NSAIDs without their unwanted side effects, most notably gastrointestinal ulceration and renal toxicity (see Patrono, this Perspective series, ref. 19). The available COX-2 inhibitors do not yet fulfill all expectations, as both nephrotoxicity (20) and gastrointestinal side effects still occur, and the latter are common, but the incidence of serious gastrointestinal complications (perforation, ulcer, bleeding) appears to be reduced (21). This experiment is still in progress per se, since many prescriptions have been filled (22) and unexpected effects of these drugs may yet emerge (23).

\section{Unorthodox routes (I): novel routes}

for prostanoid biogenesis in seminal fluid

PGs were initially discovered in human seminal fluid and originate from the seminal vesicles. Semen contains PGs derived from the three fatty acids, dihomogammalinoleic, arachidonic, and eicosapentaenoic acids. The two most abundant PGs are $19 R$-hydroxy-PGE 1 and $19 R$-hydroxy-

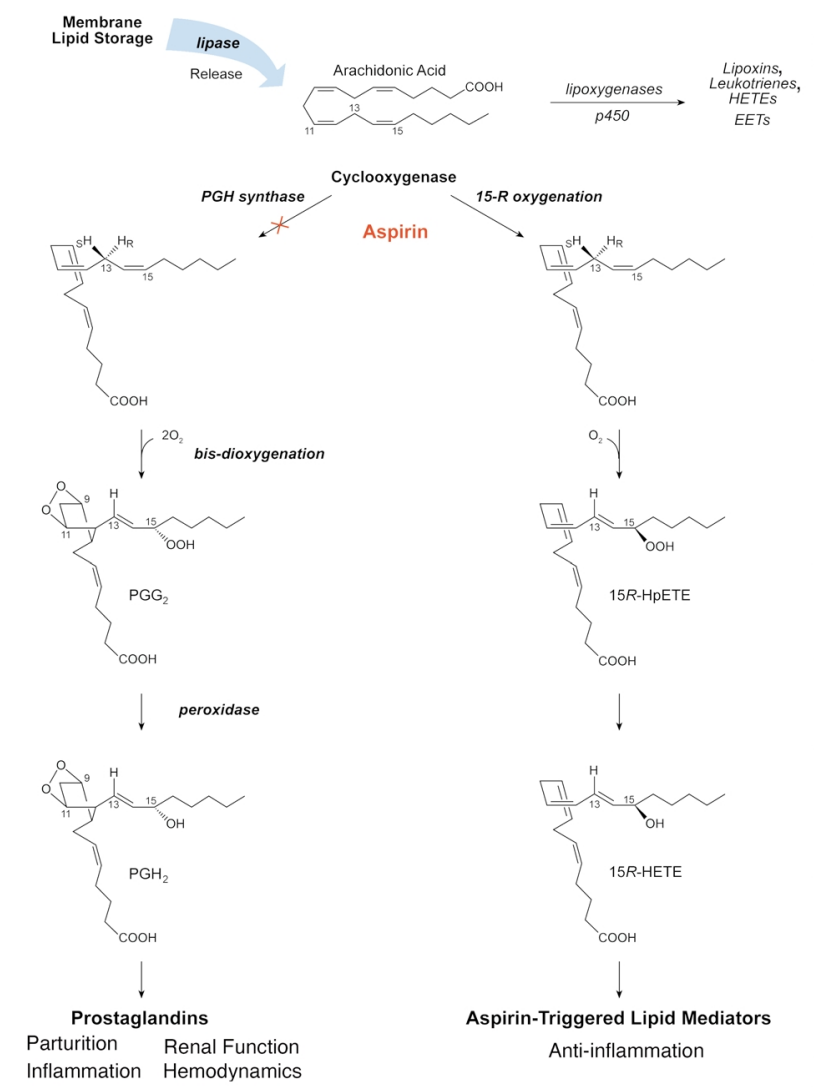

\section{Figure 1}

Cyclooxygenase reactions and products: prostaglandins and the impact of aspirin. Conversion of arachidonic acid to bioactive products involves stereoselective insertion of oxygen governed by COX (see text for details). Aspirin blocks formation of PGs from COX-1 and -2. In addition, with COX-2, aspirin triggers the formation of bioactive aspirin-triggered lipid mediators. 
$\mathrm{PGE}_{2}$ (24). Although PGE compounds are present in 100,000 -fold higher concentration in human seminal fluid $(0.4 \pm 0.1 \mathrm{mM})$ than in any other body fluids $(24,25)$, the physiological functions of seminal fluid PGEs remain a mystery, in spite of research efforts since the mid-1930s. It is puzzling that these huge amounts of PGs occur in semen, as PGs have powerful local effects on the pregnant uterus and are even used as drugs to induce cervical ripening and abortion. PGE compounds activate PGE receptors (EP receptors) (see Narumiya and FitzGerald, this Perspective series, ref. 26; and Koller et al., this series, ref. 27), but specific EP receptors for $19 R$-hydroxy-PGE compounds have not yet been discovered. 19-Hydroxy-PGE 2 is an agonist of the EP2 receptor, but less potent than $\mathrm{PGE}_{2}$ (28). Perhaps the most appealing hypothesis holds that $\mathrm{PGE}_{1}, \mathrm{PGE}_{2}, 19 R$-hydroxy-PGE ${ }_{1}$, and $19 R$-hydroxy-PGE 2 act through one or more EP receptors to cause immunosuppression within the female genital tract. These PGE compounds have powerful effects on cytokine production by antigen-presenting cells and can induce tolerance to antigens in the cervix (29). Interestingly, there is a large interindividual variation in the absolute and relative levels of PGE and 19-hydroxy-PGE in human seminal fluid. A majority of men are rapid hydroxylators, with PGE/19hydroxy-PGE ratios below 0.4. In semen of slow hydroxylators, this ratio may exceed 1.0 (25), whereas a small number of men are ultrarapid hydroxylators with PGE/19-hydroxy-PGE ratios below 0.1 .

The mechanism of biosynthesis of the 19R-hydroxyPGEs has remained unexplained since their discovery. Microsomes of human seminal vesicles and NADPH oxygenate $\mathrm{PGE}_{2}$ to 19 -hydroxy-PGE 2 very slowly compared with the efficient biosynthesis in vivo (24). Bylund et al. (4) hypothesized that seminal 19-hydroxy-PGE might be formed by one of the cytochrome P450 enzymes of the CYP4 family, which are known to act on fatty acids, LTs, and PGs. These investigators cloned and sequenced a novel member of this family from human seminal vesicles and expressed the enzyme, designated CYP4F8, in yeast in order to investigate its catalytic properties. This work showed that recombinant CYP4F8 oxidizes $\mathrm{PGE}_{2}$ only slowly and $\mathrm{PGE}_{1}$ hardly at all to their 19-hydroxy metabolites (4), but the possibility remained that $\mathrm{PGH}_{1}$ or $\mathrm{PGH}_{2}$ (see Figure 1), unstable precursors of $\mathrm{PGE}_{1}$ and $\mathrm{PGE}_{2}$, respectively, might be transformed by cytochromes $\mathrm{P} 450$. Bylund et al. addressed this possibility using several stable analogues of $\mathrm{PGH}_{2}$ and showed that recombinant CYP4F8 can indeed metabolize $\mathrm{PGH}_{1}$ and $\mathrm{PGH}_{2}$ to their 19-hydroxy-derivatives. Moreover, microsomes of human seminal vesicles, in the presence of NADPH, transformed the stable PG endoperoxide analogs and $\mathrm{PGH}_{1}$ and $\mathrm{PGH}_{2}$ in essentially the same way as did recombinant CYP4F8. Seminal vesicles contain PGE synthase, and it is likely that 19-hydroxy-PGH compounds are isomerized to 19-hydroxy-PGE compounds by PGE synthases (30). CYP4F8 is the first described hydroxylase with specificity and catalytic competence for PG endoperoxides. It will be of interest to determine whether interindividual variation in PGE/19-hydroxy-PGE ratios is due to polymorphisms in the gene for CYP4F8.

\section{Unorthodox routes (II): autooxidation} and enzymatic oxidation of fatty acids

Polyunsaturated fatty acids derived from membrane phospholipids can undergo autooxidation in vivo, generating a complex mixture of hydroperoxides, epoxides, and cyclic peroxides (31). Of particular medical interest are the isoprostanes and epoxides of arachidonate and other polyunsaturated fatty acids (PUFAs).

The isoprostanes are derived from cyclic peroxides and constitute a family of PG-related compounds with a complex chemistry. The nomenclature describing these compounds is partly based on that of PGs (32). For instance, the isoprostanes of the $\mathrm{F}_{2 \alpha}$ series retain the cyclopentane ring of $\mathrm{PGF}_{2 \alpha}$ and contain the same number of double bonds, although their side chains differ from $\mathrm{PGF}_{2 \alpha}$. Isoprostane $\mathrm{F}_{2 \alpha}$-III ( $\mathrm{iPF}_{2 \alpha}$-III, previously designated 8-iso-PGF ${ }_{2 \alpha}$ ) was first discovered in human urine. This compound serves as a marker of in vivo lipid peroxidation $(33,34)$, as do several other isoprostanes, particularly $\mathrm{iPF}_{2 \alpha}-\mathrm{I}, \mathrm{iPF}_{2 \alpha}-\mathrm{IV}$, and isomers of 8,12-iso$\mathrm{iPF}_{2 \alpha}$-IV (35-37). The isoprostane indices of lipid peroxidation increase following bolus injection of LPSs in humans (38), and they are elevated in cigarette smokers compared with controls and in patients with acute alcoholic hepatitis (39). Similarly, Tangirala et al. (40) have shown that, whereas LDL receptor-deficient mice contain large amounts of these lipids in their urine and in their atherosclerotic lesions, gene therapy with apoE cDNA can revert these biochemical phenotypes in parallel with a suppression of arterial disease. Thus, the urinary isoprostane index seems to offer a credible method to estimate lipid peroxidation in various diseases.

Isoprostanes may also mediate some aspects of oxidative stress. Several isoprostanes are formed in sufficiently large amounts that they may act as incidental ligands of prostanoid receptors, such as the $\mathrm{PGF}_{\alpha}(\mathrm{FP})$ and the $\mathrm{TXA}_{2}(\mathrm{TP})$ receptors. $\mathrm{PGF}_{2 \alpha}$ and 8,12 -iso-iPF $2 \alpha$-III activate the FP receptor of neonatal rat ventricular myocytes, which causes myocyte hypertrophy through activation of different signal transduction pathways (41). In human platelets, $\mathrm{iPF}_{2 \alpha}$-III induces shape change, calcium mobilization, reversible aggregation, and inositol phosphate formation. The latter effect cannot be blocked by TP receptor antagonists, suggesting that $\mathrm{iPF}_{2 \alpha}-\mathrm{III}$ acts on platelets through routes other than TP receptor activation (42). These observations suggest the intriguing possibility that isoprostanes act as autacoids, but evaluating their significance will require further studies (32).

Epoxides of PUFAs can be formed by autooxidation, by cytochrome $\mathrm{P} 450$, and possibly by the oxidative burst of inflammatory cells $(31,43)$. Epoxides of linoleic acid 
Prostaglandins and their precursors

Garret A. FitzGerald, Series Editor

are toxic, whereas epoxides of arachidonic acid (EETs) have a wide range of biological effects (see ref. 44 for recent review). The 5,6-epoxide of arachidonic acid $(5,6$-EET) provides an unorthodox route to oxidized prostanoids. 5,6-EET is an excellent substrate of COX and thromboxane synthase, which transform 5,6-EET to 5,6-epoxyprostaglandins (45) and 5,6-epoxythromboxanes (46). Vascular and renal effects of 5,6-EET can be blocked by inhibitors of COX enzymes (47), which suggests that the pharmacological effects of 5,6-EET can result from its transformation by COX. Distinct receptors for epoxyprostanoids have not been described, and it seems likely that both epoxyprostaglandins and isoprostanes are incidental ligands of prostanoid receptors, which may mediate some of their effects.

Unorthodox routes (III):

aspirin-triggered lipid mediators

Despite about 100 years of wide use, ASA's therapeutic impact is still evolving, and new beneficial effects are still being uncovered $(48,49)$. The irreversible acetylation of both COX-1 and COX-2 with subsequent inhibition of PG is well appreciated and explains some but not all of ASA's pharmacological actions (7). Until recently, the mechanism for ASA impact in vivo on polymorphonuclear leukocyte (PMN) recruitment in inflammation remained largely unknown. In 1995, Clària and Serhan (2) reported that ASA triggers formation of novel series of lipid mediators, the aspirintriggered lipoxins (ATL), whose formation relies on cellcell interactions (15-epi-LX; Figure 1, right, and Figure 2). Coactivation of neutrophils and certain epithelial cells treated with ASA generates a novel class of $15 R-$ containing LXs termed 15-epi-LX (ATL), which downregulate $\mathrm{PMN}$-endothelial cell interactions and epithelial cell function $(50,51)$.

In most clinical arenas ASA is believed to act in lipid metabolism strictly as an inhibitor of COX function. However, when ASA inhibits PG, the ASA-acetylated form of COX-2 is still active and converts arachidonate to 15 -HETE that carries its $\mathrm{C} 15$ alcohol in the $R$ configuration $(2,52)$. This is the result of an unusual L-shaped binding of arachidonic acid within the COX-2 substrate channel (9), which is larger in this isoenzyme (see recent crystal structures for COX-1 [ref. 10] and COX-2 [ref. 53]). The ATL 15-epi-LXA 4 is more potent and longer acting than the native, $15 \mathrm{~S}$-containing LX, as it is not as rapidly metabolically inactivated (50). Hence, it appears that ASA triggers formation of endogenous eicosanoids and related substances, which may mediate some of its beneficial actions by pirating the native pathway of LX signaling. Crucially, the biosynthesis of 15-epi-LXs does not arise from a metabolic shunt but rather represents the effect of ASA on the oxygenating function of COX-2 in situ at foci of inflammation.
The active site of COX-2 appears to be more flexible than COX-1 (10). While the normal biological importance of this difference is not clear, the presence of an additional binding pocket in COX-2 for NSAID was reportedly exploited to produce COX-2-specific inhibitors (10). Acetylation of COX-1 by ASA does not permit substantial amounts of arachidonate conversion to $15 R$-HETE - yet another clear difference between these two otherwise highly conserved enzymes, since COX-2-ASA apparently carries out this process efficiently.

Newly synthesized ASA analogs have been introduced that display potent anti-inflammatory properties and also permit $15 R$-HETE generation by COX-2 (54). Once formed, $15 R$-HETE is rapidly esterified in inflammatory cells, altering signal transduction as well as priming the supply of LX precursors (55). The endothelial cell production of 15-HETE is highly effective in situ (3) and in vivo at sites of inflammation (56). Given the vast size

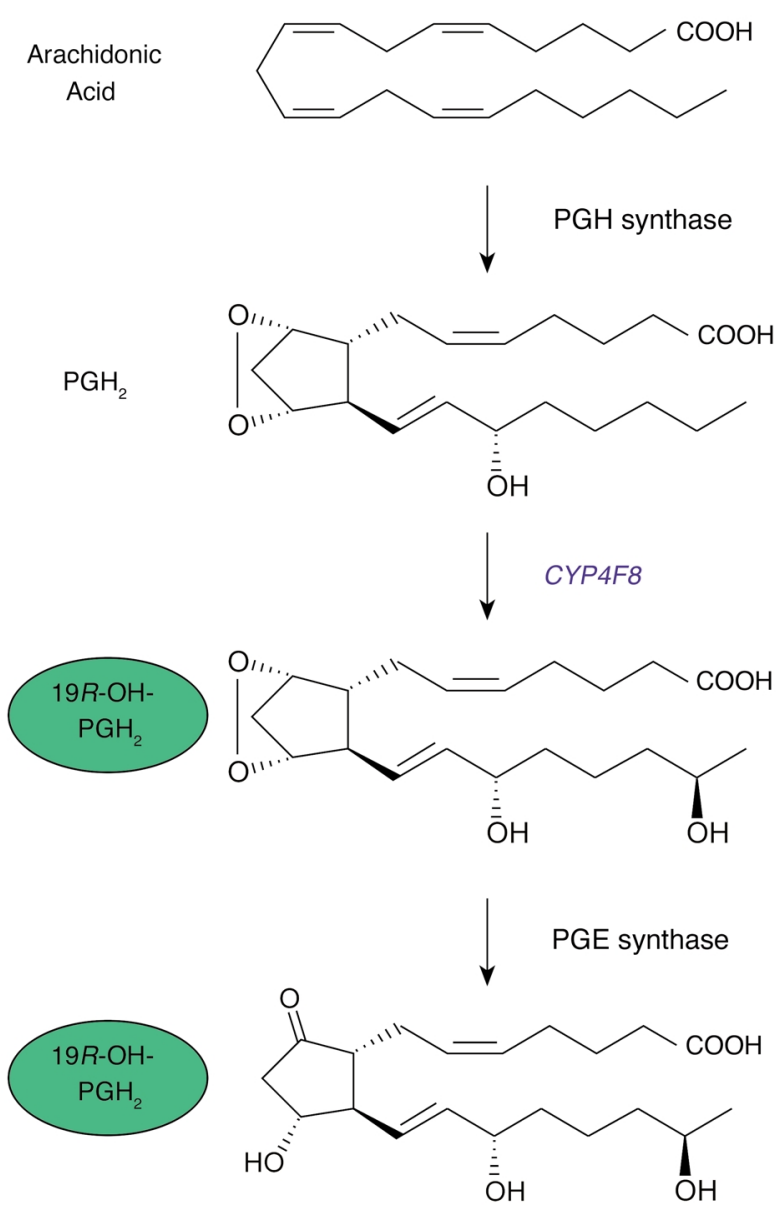

Figure 2

Biosynthesis of $19 R$-hydroxy-PGE from $\mathrm{PGH}_{2}$ by CYP4F8 and PGE synthases. $19 R$-hydroxy-PGE is a major eicosanoid in humans. This novel biosynthesis route indicates that the 19 position $R$ alcohol is in place before the $\mathrm{PGE}_{2}$ ring is made (see text). 
of the vasculature and its role in host defense and inflammation, the vascular endothelium is likely to contain focal regions or "hot spots" under stress that express COX-2 and can generate substantial amounts of COX-2-derived products with ASA treatment. Because COX 2 polymorphisms are found in approximately $5 \%$ of the population (18), structural variants of this enzyme might be found in the population that could affect an individual's ability to generate $15 R$-HETE without ASA treatment or that alter the enzyme's sensitivity to NSAIDs and its ability to produce these new products.

\section{ATLs, LXs, and the resolution of inflammation}

Cell-cell interactions and transcellular biosynthesis of mediators are now well recognized as important means of generating new lipid mediators (57). In humans, LX biosynthesis is an example of lipoxygenase-lipoxygenase (LO-LO) interactions via transcellular circuits. Thus, LXs are generated by two main routes. The first described involves initial lipoxygenation by $15-\mathrm{LO}$ (inserting $\mathrm{O}_{2}$ in predominantly the $S$ configuration) followed by 5 -LO. This route is particularly relevant whenever PMNs interact at mucosal surfaces. A second route, which occurs predominantly within the vasculature when platelet glutathione is depleted, involves the 5-LO-mediated release of $\mathrm{LTA}_{4}$ from leukocytes and the subsequent conversion of this compound to LXs by platelets. Human platelets alone do not generate LX on their own but become an important source as a result of their interactions with PMNs (55).

In human disease, LXs are generated in airway, kidney, joints, liver, and vessels (ref. 58; see also review, ref. 55 and references therein), and their actions stand apart from other known lipid mediators. Acting in the nanomolar range, native LXs selectively regulate the motility of PMNs, eosinophils, and monocytes in a stereospecific fashion, which raised the possibility that these compounds serve as endogenous "stop signals" by which these leukocytes help resolve local inflammation (55). Much as LXs serve as endogenous suppressors of inflammation, the ATL 15-epi-LX (stereoisomers of native LX) and related compounds may be the effectors of well-established anti-inflammatory therapies. Furthermore, as Chavis et al. (59) recently suggested, LXs themselves may mediate the anti-inflammatory cellular actions of glucocorticoids.

LXs are rapidly generated, act locally, and are quickly inactivated. Based on knowledge of LX routes of inactivation and the identity of a receptor for $\mathrm{LXA}_{4}$ (60), stable LX and ATL analogs have been designed that resist rapid metabolic inactivation and regulate leukocyte traffic. These compounds are also topically active inhibitors of acute inflammation (Figure 2 and Table 1) and have proved to be potent inhibitors of $\mathrm{TNF}_{\alpha}$ signals and IL-8 formation (51). In addition, ATL and its active analogs compete with $\mathrm{LXA}_{4}$ at its own receptor on leukocytes, acting as an agonist to induce "stop signaling" (61). These results support the notion that ATL and native LX prevent damage by serving as endogenous anti-inflammatory molecules.

\section{Novel anti-inflammatory pathways}

for $\omega-3$ fish oil and COX-2

Over the past 25 years, numerous reports have suggested that dietary supplementation with $\omega-3$ polyunsaturated fatty acids ( $\omega$-3 PUFAs) has beneficial effects in human disease. Recent reviews discuss potential antithrombotic, immunoregulatory, and anti-inflammatory responses relevant in arteriosclerosis, arthritis, and asthma, as well as antitumor and antimetastatic effects (62). The possible preventative or therapeutic actions of $\omega-3$ PUFA supplementation in infant nutrition, cardiovascular diseases, and mental health led an international workshop to a call for recommended dietary intakes (63). Data from one large trial (GISSI-Prevenzione, which included more than 11,300 subjects), which evaluated the benefits of aspirin with or without $\omega-3$ PUFA supplementation for patients surviving myocardial infarction, found a significant decrease in cardiovascular death in the group taking the supplement (64).

Fish oil has been proposed to act by one or more of several mechanisms. First, it may prevent conversion of arachidonic acid to proinflammatory eicosanoids, such as PGs and LTs. Second, it may provide an alternative substrate for the 5-LO, promoting the production of

\section{Table 1}

Anti-inflammatory and resolving actions of novel aspirin-triggered lipid mediators

\begin{tabular}{|c|c|}
\hline ATLMs & Response/Action \\
\hline $\begin{array}{l}\mathrm{LXA}_{4}, \mathrm{ATL} \\
\text { and stable analogs }\end{array}$ & $\begin{array}{l}\text { Interact with specific receptors on leukocytes, neutrophils, } \\
\text { eosinophils, and monocytes, regulating their function }\end{array}$ \\
\hline LX, ATL, and analogs & $\begin{array}{l}\text { Inhibit PMN-mediated inflammation } \\
\text { in skin, lung, and kidney in murine models }\end{array}$ \\
\hline ATL & Protect in reperfusion injury \\
\hline $\mathrm{LXA}_{4}, \mathrm{ATL}$, and analogs & Enhance macrophage phagocytosis of apoptotic leukocytes \\
\hline $\mathrm{LXA}_{4}, \mathrm{ATL}$, and analogs & $\begin{array}{l}\text { Redirect chemokine and cytokine expression and gene regulation } \\
\text { (IL- } 4 \uparrow, \text { IL-1 } 1 \beta \downarrow, I L-8 \downarrow) \text {, and block TNF- } \alpha \text { actions }\end{array}$ \\
\hline \multirow[t]{3}{*}{$\mathrm{LXA}_{4}$ and $\mathrm{ATL}$ analogs } & Enhance clearance and accelerate resolution of pulmonary edema \\
\hline & Reduce COX-2 traffic in pain responses \\
\hline & Antiangiogenic properties \\
\hline $18 R$ series $(18 R, 5,12-\mathrm{THEPE})$ & Inhibits PMN transmigration and anti-inflammation \\
\hline
\end{tabular}
and 15 -epi-LXA $A_{5}$ series

ATL, aspirin-triggered lipoxin, 15R-LXA 4 ; ATLM, aspirin-triggered lipid mediator. For further details, see text. Full references appear in the suggested reading list (http://www.jci.org/cgi/content/full/107/12/1481/DC1). 
Prostaglandins and their precursors

Garret A. FitzGerald, Series Editor

the less potent 5 -series LTs, rather than the potent 4series or arachidonic acid-derived LTs. Third, it might be converted by $\mathrm{COX}$ to $\mathrm{PGI}_{3}$ or related 3-series prostanoids, which are antithrombotic agents (62). Neither these nor other proposed explanations are widely accepted, largely because of the suprapharmacologic amounts of $\omega-3$ PUFA that are required in vitro to achieve the supposed beneficial actions and because compelling molecular evidence has been lacking. Nevertheless, in view of qualitatively similar beneficial profiles attributed to dietary $\omega-3$ PUFA and to aspirin in a variety of diseases, several groups have sought evidence for possible new lipid-derived signals that could explain the epidemiological findings.

Vascular COX-2 and microinflammation:

ASA and $\omega-3$ PUFA treatment

Following administration of $\omega-3$ and ASA, inflammatory exudates formed in murine dorsal pouches generate several novel compounds (Figure 3), including 18R-hydroxy-eicosapentaenoic acid (18R-HEPE) and several trihydroxy-containing compounds derived from fish oil EPA (C20:5). Human cells also generate these new $18 \mathrm{R}$ and $15 \mathrm{R}$ series of compounds from EPA, which have proved to carry intriguing bioactivities. When human endothelial cells expressing COX-2 are pulsed with EPA and treated with ASA, they generate either 18R-HEPE or a mixture of 18RHEPE and 15R-HEPE, depending on the source of the cells. The involvement of COX-2 in this biosynthetic pathway is supported by in vitro results obtained with recombinant human COX-2, in which the enzyme was exposed to ASA and $\omega-3$ PUFA. Acetylation of COX-2 by ASA dramatically increased the production of both 18R-HEPE and 15R-HEPE. Acetylated COX-2 in endothelial cells (Figure 3 ) is a dominant source of 18R-HEPE and 15R-HEPE, findings that may be of clinical significance (3).
Trout macrophages and human leukocytes can indeed convert endogenous EPA to 15S-containing LX or 5 -series $\mathrm{LX}_{5}(65)$. When engaged in phagocytosis, activated human PMNs process the intermediates derived from acetylated recombinant COX-2 to produce two series of trihydroxy-containing EPE, one carrying an $18 R$-hydroxyl group, and the other related to

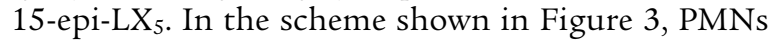
take up $18 R$-HEPE and convert it via a 5-lipoxygenation to insert molecular oxygen and, in subsequent steps, form 5-hydro(peroxy)-18R-DiH(p)EPE and an unstable 5(6)epoxide that gives rise to 5,12,18R-triHEPE. In an analogous biosynthetic pathway, $15 R$-HEPE released by endothelial cells is converted by activated PMN via 5-lipoxygenation to a 5-series $\mathrm{LXA}_{5}$ analog (Figure 3) that also retains their C15 $R$ or epi

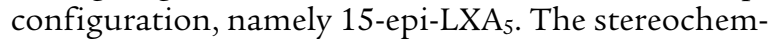
istry of compounds in this pathway is different from that of the compounds of the LO-LO-driven pathways, which yield predominantly $\mathrm{C} 15 \mathrm{~S}$-containing $\mathrm{LX}_{5}$ structures (members of the so-called 5-series) as observed from endogenous sources of EPA in trout macrophages (ref. 65 and references therein). With ASA-COX-2, the chirality of the precursor $15 R$ is retained by human PMNs to give 15 -epi-LXA 5 (Figure 3 ), the 5-series $\omega$-3 analog of 15-epi-LXA 4 . As also observed in LX biosynthesis initiated by $15-\mathrm{LO}$, conversion of both $18 R$ - and $15 R$-HEPE by activated PMNs with 5-lipoxygenation is accompanied by a reduction in LT formation in these cells (3).

The anti-inflammatory impact of COX-2-initiated pathways

Since a well-recognized locus of action for traditional anti-inflammatory therapies is PMN transendothelial migration, the identification of endogenous lipid mediators that can control these cell-cell interactions (3) and serve as anti-inflammatory agonists is of considerable

\section{Figure 3}

Aspirin-triggered lipid mediators: biosynthesis of 15-epi-LXs. Irreversible acetylation of COX-2 by aspirin changes the enzyme's product from PGs intermediate to precursors of ATLMs. The acetylated COX-2 remains catalytically active (see text).

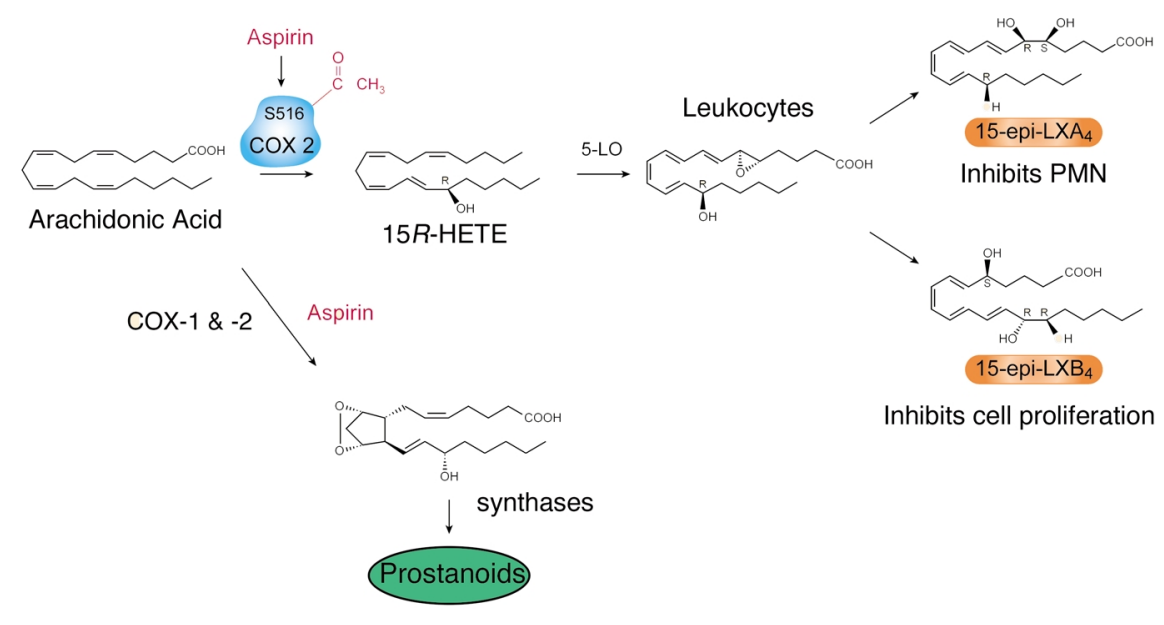


interest. In this regard, both 5,12,18R-triHEPE and its precursor $18 R$-HEPE prove to be inhibitors of $\mathrm{LTB}_{4}$-stimulated PMN transendothelial migration. Hence, the new $18 R$-series members might serve as a damper for inflammatory responses within the vascular microenvironment. The novel 18R-HEPE shows some inhibitory activity, and its product 5,12,18R-triHEPE potently inhibits PMN infiltration in vivo. In addition, commonly used drugs such as indomethacin and ASA could still permit PUFA oxygenation by activated endothelial cells (3). Hence, it will be of interest to determine whether direct enzymatic oxygenation by COX-2-NSAID complexes can indeed occur at sites of vascular inflammation in human disease. These findings raise the question of whether arachidonate is the sole physiologically relevant substrate for COX-2 in the human tissues or whether EPA or other PUFAs are important as well (3). Despite the many reports of possible beneficial impact of $\omega$-3 PUFAs and EPA in humans $(62-64,66)$, oxygenation by COX-2 to generate similar bioactive compounds has not been addressed. In fish, both EPA (C20:5) and arachidonic acid are mobilized in macrophages and platelets to produce 5-series and 4-series eicosanoids (including PG, LT, and LX) with approximately equal abundance (65). In humans, given the gram amounts of $\omega-3$ PUFA taken as dietary supplements (see refs. 62-64) and the large area of the vasculature that can express COX-2, the conversion of EPA by endothelial cells and neighboring cells at vascular hot spots of local inflammation (Figure 3) could represent a significant source in vivo. The COX-2-dependent conversions of $\omega$-3 PUFA are likely to be elevated within inflamed or diseased tissues, where COX-2 is highly expressed and upregulated. Such local effects on fatty acid metabolism (56) may greatly influence the therapeutic benefit of ASA treatment.

Analogous to 15-epi-LX biosynthesis, COX-2-derived $15 R$-HEPE is converted by 5 -lipoxygenation with 5(6) epoxide formation in leukocytes to give the 15-epi-LX series (Figure 3 ). The stable analogs of 15 -epi-LXA ified with bulky groups at their $\mathrm{C}-15$ position through position C-20, resist inactivating enzymes and are more potent in vivo, inhibiting PMN traffic as well as suppressing the formation and actions of key proinflammatory cytokines $(50,67)$. These new 5-series 15 -epi-LX are likely to act in a fashion similar to that of the synthetic analogs ATL and LX analogs because they possess a $\Delta 17$ double bond and could thus serve as $\omega$-3-derived 15-epi-LX-like analogs. Since COX-2-ASA-dependent oxygenation (Figures 3 and 4) produces bioactive compounds in vivo that block PMN transendothelial migration and infiltration, the findings provide a basis for a novel mechanism of action for this NSAID and dietary $\omega-3$ supplementation: namely, the generation of endogenous functional arrays of lipid signals that could dampen key events in microinflammation (3) and thereby account for some of the beneficial actions noted for $\omega-3$ therapies in human trials.

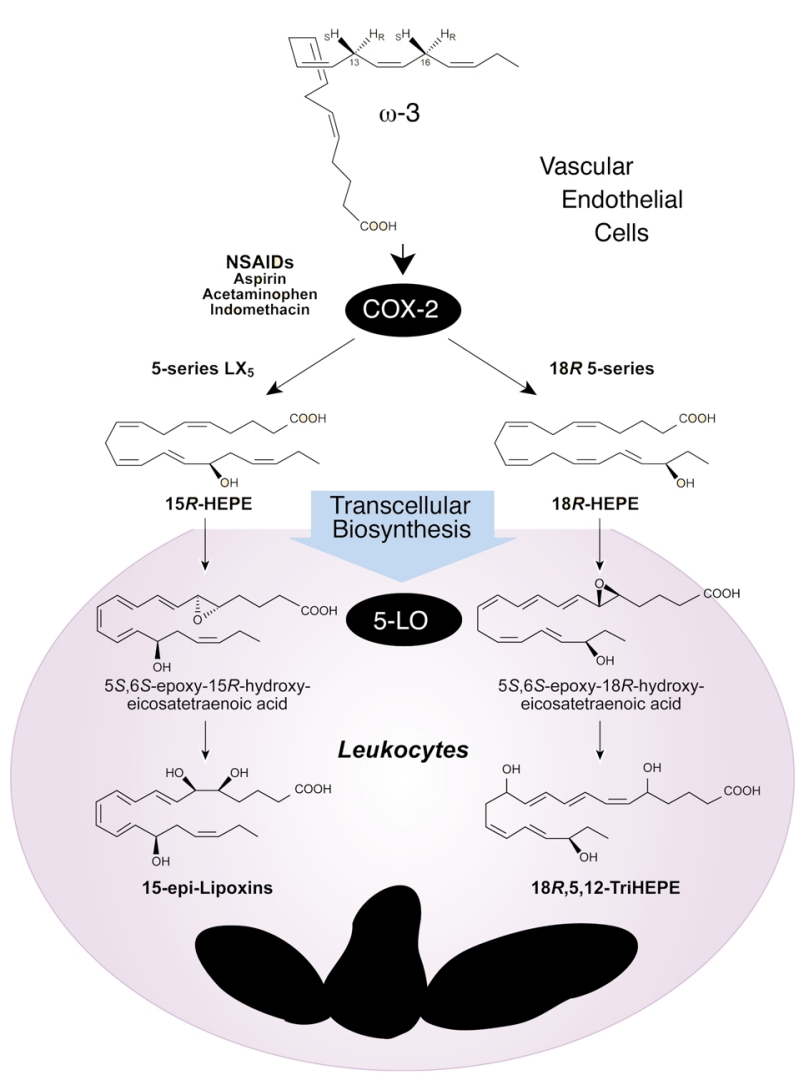

Figure 4

Functional arrays of lipid signals generated via transcellular processing $\omega$ 3 PUFA: endogenous inhibitors of microinflammation. At sites where COX2 is expressed and treated with NSAIDs, although PG formation is blocked, systemic $\omega-3$ PUFA can be converted via a COX-2-NSAID-dependent LOtype mechanism (stereospecific hydrogen abstraction at $\mathrm{C} 16$ or $\mathrm{C} 13$ in C20:5 to give $R$ insertions of molecular $\mathrm{O}_{2}$ ) to yield $15 R-\mathrm{H}(\mathrm{p}) \mathrm{EPE}$ or $18 R-\mathrm{H}(\mathrm{P}) \mathrm{EPE}$. These compounds are reduced to alcohols and converted to epoxide intermediates. Elucidation of the complete stereochemistry of the trihydroxy-compounds is in progress, and they are depicted in their likely configuration. The products interact with cells in the local microenvironment, inhibiting PMN recruitment. See text and suggested reading list (http://www.jci.org/cgi/content/full/107/12/1481/DC1) for details.

\section{Concluding remarks}

Inappropriate inflammation is now recognized to contribute to cardiovascular disease (49) and to other clinical syndromes with PMN-mediated tissue injury. Elucidation of the novel $\omega$-3 PUFA processing pathways evoked during cell-cell interactions and the unexpected impact of NSAIDs in vascular inflammatory exudates (Figures 3 and 4) opens new possibilities to rationalize earlier dietary (i.e., fish oil-based) supplementation (as in ref. 64) as well as mechanisms to generate potent local endogenous mediators that control microinflammation (3). Moreover, since docosahexaenoic acid C22:6, another major $\omega$-3 PUFA in fish oil, inhibits COX-1 (68), these new pathways and products (Figure 
Prostaglandins and their precursors Garret A. FitzGerald, Series Editor

4) offer a basis for endogenous regulation to assuage microinflammation. Controlling COX-1 and COX-2 pathways by these means may also circumvent unwanted effects of current anti-inflammatory therapies.

Each day many succumb to diseases characterized by excessive inflammation-related events. Aspirin as well as other NSAIDs that affect these signaling systems (Figures 1 and 2) are in wide use, yet these agents are not without harmful side effects, particularly in the kidney and stomach. The discovery of the second isoform of COX (reviewed in ref. 15) sparked a large-scale search for safer aspirin-like drugs, namely selective COX-2 inhibitors, that would bypass the unwanted side effects of steroid or NSAID treatment (69). Results reviewed here indicate that LXs, their aspirin-triggered epimers (ATL), and broader arrays of aspirin-triggered lipid mediators derived from $\omega-3$ PUFA (Figure 3 ) reveal previously unappreciated endogenous anti-inflammatory mechanisms (Table 1) that could offer a new approach to treatment. Since ASA is a well-tolerated treatment by most, it is essential to fully elucidate its mechanisms of action to improve its use.

The finding that LX counters inflammatory events led to more general concepts, namely that aspirin-triggered lipid mediators could serve as local mediators of antiinflammation or endogenous agonists that favor resolution of inflammation. Additional support for the notion that LXs and aspirin-triggered lipid mediators are protective comes from the recent findings that $\mathrm{LXA}_{4}$ stimulates macrophages to clear apoptotic PMNs (70), and that $\mathrm{LXA}_{4}$ receptors regulate gene expression or cytokines and metalloproteases (see ref. 71 and references therein). This work adds a new dimension to the well-established use of low-dose ASA as a specific COX1 inhibitor in platelets, where the primary COX product, $\mathrm{PGH}_{2}$, is a key intermediate. We now know that $\mathrm{PGH}_{2}$ can be metabolized by CYP4F8 to 19-hydroxy PG endoperoxides, which are the immediate precursors of 19-hydroxy-PGE 1 and 19-hydroxy-PGE $\mathrm{P}_{2}$ in human seminal fluid, and likely of reproductive importance. The transformation of $\mathrm{PGH}_{2}$ by cytochromes $\mathrm{P} 450$ in different tissues and disease states has not yet been fully investigated. In view of the results reviewed here, it is likely that $\mathrm{PGH}_{2}$ will engage in transcellular biosynthesis. These unorthodox pathways open new avenues and provide an unexpected view of $\mathrm{PGH}_{2}$ and the ASA-triggered novel lipid mediators that are of interest both in the reproductive system and the study of endogenous control of inflammation and tissue injury.

\section{Acknowledgments}

These studies were supported in part by NIH grants GM38765 and P01-DE13499 (to C.N. Serhan) and the Swedish Medical Research Council (03X-06523). Apologies to our colleagues whose reviews were cited rather than original references because of space limitations. Addi- tional references can be found in the suggested reading list at http://www.jci.org/cgi/content/full/107/12/ 1481/DC1.

1. von Euler, U.S. 1936. On the specific vaso-dilating and plain muscle stimulating substances from accessory genital glands in man and certain animals (prostaglandin and vesiglandin). J. Physiol. 88:213-234.

2. Clària, J., and Serhan, C.N. 1995. Aspirin triggers previously undescribed bioactive eicosanoids by human endothelial cell-leukocyte interactions. Proc. Natl. Acad. Sci. USA. 92:9475-9479.

3. Serhan, C.N., et al. 2000. Novel functional arrays of lipid-derived mediators with antiinflammatory actions generated from omega- 3 fatty acids via cyclooxygenase 2-nonsteroidal antiinflammatory drugs and transcellular processing. J. Exp. Med. 192:1197-1204.

4. Bylund, J., Hidestrand, M., Ingelman-Sundberg, M., and Oliw, E.H. 2000. Identification of CYP4F8 in human seminal vesicles as a prominent 19- hydroxylase of prostaglandin endoperoxides. J. Biol. Chem. 275:21844-21849.

5. Bergström, S. 1982. The prostaglandins: from the laboratory to the clinic. In Les prix nobel: Nobel prizes, presentations, biographies and lectures. Edited by The Nobel Foundation. Almqvist \& Wiksell. Stockholm, Sweden. 129-148.

6. Samuelsson, B. 1982. From studies of biochemical mechanisms to novel biological mediators: prostaglandin endoperoxides, thromboxanes and leukotrienes. In Les prix nobel: Nobel prizes, presentations, biographies and lectures. Edited by The Nobel Foundation. Almqvist \& Wiksell. Stockholm, Sweden. 153-174.

7. Vane, J.R. 1982. Adventures and excursions in bioassay: the stepping stones to prostacyclin. In Les prix nobel: Nobel prizes, presentations, biographies and lectures. Edited by The Nobel Foundation. Almqvist \& Wiksell. Stockholm, Sweden. 181-206.

8. Samuelsson, B. 1965. On the incorporation of oxygen in the conversion of 8,11,14-eicosatrienoic acid to prostaglandin E1. J. Am. Chem. Soc. 87:3011-3013.

9. Rowlinson, S.W., et al. 2000. Spatial requirements for 15-(R)-hydroxy$5 Z, 8 Z, 11 Z, 13 E$-eicosatetraenoic acid synthesis within the cyclooxygenase active site of murine COX-2. J. Biol. Chem. 275:6586-6591.

10. Malkowski, M.G., Ginell, S.L., Smith, W.L., and Garavito, R.M. 2000. The productive conformation of arachidonic acid bound to prostaglandin synthase. Science. 289:1933-1937.

11. Fitzpatrick, F.A., and Soberman, R. 2001. Regulated formation of eicosanoids. J. Clin. Invest. 107:1347-1351.

12. Xiao, G., et al. 1997. Analysis of hydroperoxide-induced tyrosyl radicals and lipoxygenase activity in aspirin-treated human prostaglandin $\mathrm{H}$ synthase-2. Biochemistry. 36:1836-1845.

13. Oliw, E.H., Hornsten, L., Sprecher, H., and Hamberg, M. 1993. Oxygenation of 5,8,11-eicosatrienoic acid by prostaglandin endoperoxide synthase and by cytochrome P450 monooxygenase: structure and mechanism of formation of major metabolites. Arch. Biochem. Biophys. 305:288-297.

14. Yu, M., Ives, D., and Ramesha, C.S. 1997. Synthesis of prostaglandin E2 ethanolamide from anandamide by cyclooxygenase-2. J. Biol. Chem. 272:21181-21186.

15. Kozak, K.R., Rowlinson, S.W., and Marnett, L.J. 2000. Oxygenation of the endocannabinoid, 2-arachidonylglycerol, to glyceryl prostaglandins by cyclooxygenase-2. J. Biol. Chem. 275:33744-33749.

16. Herschman, H.R. 1998. Recent progress in the cellular and molecular biology of prostaglandin synthesis. Trends Cardiovasc. Med. 8:145-150.

17. Smith, W.L., and Langenbach, R. 2001. Why there are two cyclooxygenase isozymes. J. Clin. Invest. 107:1491-1495.

18. Jones, D.A., Carlton, D.P., McIntyre, T.M., Zimmerman, G.A., and Prescott, S.M. 1993. Molecular cloning of human prostaglandin endoperoxide synthase type II and demonstration of expression in response to cytokines. J. Biol. Chem. 268:9049-9054.

19. Patrono, C., Patrignani, P., and Rodríguez, L.A.G. 2001. Cyclooxygenaseselective inhibition of prostanoid formation: transducing biochemical selectivity into clinical read-outs. J. Clin. Invest. In Press.

20. Swan, S.K., et al. 2000. Effect of cyclooxygenase-2 inhibition on renal function in elderly persons receiving a low-salt diet. A randomized, controlled trial. Ann. Intern. Med. 133:1-9.

21. Bombardier, C., et al. 2000. Comparison of upper gastrointestinal toxicity of rofecoxib and naproxen in patients with rheumatoid arthritis. $N$. Engl.J. Med. 343:1520-1528.

22. Noble, S.L., King, D.S., and Olutade, J.I. 2000. Cyclooxygenase-2 enzyme 
inhibitors: place in therapy. Am. Fam. Physician. 61:3669-3676.

23. Schnitzer, T.J. 2001. Cyclooxygenase-2-specific inhibitors: are they safe? Am. J. Med. 110:(Suppl. 1):S46-S49.

24. Taylor, P.L., and Kelly, R.W. 1974. 19-hydroxylated E prostaglandins as the major prostaglandins of human semen. Nature. 250:665-667.

25. Oliw, E.H., and Johnsen, O. 1988. Rapid and slow hydroxylators of seminal E prostaglandins. Biochim. Biophys. Acta. 963:295-301.

26. Narumiya, S., and FitzGerald, G.A. 2001. Genetic and pharmacological analysis of prostanoid receptor function. J. Clin. Invest. In press.

27. Tilley, S.L., Coffman, T.M., and Koller, B.H. 2001. Mixed messages: modulation of inflammation and immune responses by prostaglandins and thromboxanes. J. Clin. Invest. In press.

28. Woodward, D.F., Protzman, C.E., Krauss, A.H., and Williams, L.S. 1993. Identification of 19 (R)-OH prostaglandin E2 as a selective prostanoid EP2-receptor agonist. Prostaglandins. 46:371-383.

29. Denison, F.C., Calder, A.A., and Kelly, R.W. 1999. The action of prostaglandin $\mathrm{E} 2$ on the human cervix: stimulation of interleukin 8 and inhibition of secretory leukocyte protease inhibitor. Am.J. Obstet. Gynecol. 180:614-620.

30. Jakobsson, P.J., Thoren, S., Morgenstern, R., and Samuelsson, B. 1999 Identification of human prostaglandin E synthase: a microsomal, glutathione-dependent, inducible enzyme, constituting a potential novel drug target. Proc. Natl. Acad. Sci. USA. 96:7220-7225.

31. Porter, N.A., Caldwell, S.E., and Mills, K.A. 1995. Mechanisms of free radical oxidation of unsaturated lipids. Lipids. 30:277-290.

32. Lawson, J.A., Rokach, J., and FitzGerald, G.A. 1999. Isoprostanes: formation, analysis and use as indices of lipid peroxidation in vivo. J. Biol. Chem. 274:24441-24444.

33. Roberts, L.J., and Morrow, J.D. 2000. Measurement of F(2)-isoprostanes as an index of oxidative stress in vivo. Free Radic. Biol. Med. 28:505-513

34. Morrow, J.D. 2000. The isoprostanes: their quantification as an index of oxidant stress status in vivo. Drug Metab. Rev. 32:377-385.

35. Pratico, D., et al. 1998. IPF2alpha-I: an index of lipid peroxidation in humans. Proc. Natl. Acad. Sci. USA. 95:3449-3454.

36. Lawson, J.A., et al. 1998. Identification of two major F2 isoprostanes, 8,12-iso- and 5-epi-8, 12-iso-isoprostane F2alpha-VI, in human urine. J. Biol. Chem. 273:29295-29301.

37. Li, H., et al. 1999. Quantitative high performance liquid chromatography/tandem mass spectrometric analysis of the four classes of $\mathrm{F}(2)$-isoprostanes in human urine. Proc. Natl. Acad. Sci. USA. 96:13381-13386.

38. McAdam, B.F., et al. 2000. Effect of regulated expression of human cyclooxygenase isoforms on eicosanoid and isoeicosanoid production in inflammation. J. Clin. Invest. 105:1473-1482.

39. Meagher, E.A., et al. 1999. Alcohol-induced generation of lipid peroxidation products in humans. J. Clin. Invest. 104:805-813.

40. Tangirala, R.K., et al. 2001. Reduction of isoprostanes and regression of advanced atherosclerosis by apolipoprotein E. J. Biol. Chem. 276:261-266.

41. Kunapuli, P., Lawson, J.A., Rokach, J.A., Meinkoth, J.L., and FitzGerald, G.A. 1998. Prostaglandin F2alpha (PGF2alpha) and the isoprostane, 8, 12-iso-isoprostane F2alpha-III, induce cardiomyocyte hypertrophy. Differential activation of downstream signaling pathways. J. Biol. Chem. 273:22442-22452

42. Habib, A., FitzGerald, G.A., and Maclouf, J. 1999. Phosphorylation of the thromboxane receptor alpha, the predominant isoform expressed in human platelets. J. Biol. Chem. 274:2645-2651.

43. Bylund, J., Kunz, T., Valmsen, K., and Oliw, E.H. 1998. Cytochromes $\mathrm{P} 450$ with bisallylic hydroxylation activity on arachidonic and linoleic acids studied with human recombinant enzymes and with human and rat liver microsomes. J. Pharmacol. Exp. Ther. 284:51-60.

44. Capdevila, J.H., Falck, J.R., and Harris, R.C. 2000. Cytochrome P450 and arachidonic acid bioactivation. Molecular and functional properties of the arachidonate monooxygenase. J. Lipid Res. 41:163-181.

45. Oliw, E.H. 1984. Metabolism of 5(6)oxidoeicosatrienoic acid by ram seminal vesicles. Formation of two stereoisomers of 5-hydroxyprostaglandin I1. J. Biol. Chem. 259:2716-2721.

46. Balazy, M. 1991. Metabolism of 5,6-epoxyeicosatrienoic acid by the human platelet. Formation of novel thromboxane analogs. J. Biol. Chem. 266:23561-23567.

47. Carroll, M.A., Balazy, M., Margiotta, P., Falck, J.R., and McGiff, J.C. 1993 Renal vasodilator activity of 5,6-epoxyeicosatrienoic acid depends upon conversion by cyclooxygenase and release of prostaglandins. J. Biol. Chem. 268:12260-12266

48. Cronstein, B.N., Montesinos, M.C., and Weissmann, G. 1999. Salicylates and sulfasalazine, but not glucocorticoids, inhibit leukocyte accumulation by an adenosine-dependent mechanism that is independent of inhibition of prostaglandin synthesis and 105 of NFKB. Proc. Natl. Acad. Sci. USA. 96:6377-6381.

49. Ridker, P.M., Cushman, M., Stampfer, M.J., Tracy, R.P., and Hennekens, C.H. 1997. Inflammation, aspirin, and the risk of cardiovascular disease in apparently healthy men. N. Engl. J. Med. 336:973-979.

50. Serhan, C.N., et al. 1995. Design of lipoxin $\mathrm{A}_{4}$ stable analogs that block transmigration and adhesion of human neutrophils. Biochemistry. 34:14609-14615.

51. Gewirtz, A.T., et al. 1998. Pathogen-induced chemokine secretion from model intestinal epithelium is inhibited by lipoxin $\mathrm{A}_{4}$ analogs. J. Clin. Invest. 101:1860-1869.

52. Mancini, J.A., et al. 1997. Altered sensitivity of aspirin-acetylated prostaglandin $\mathrm{G} / \mathrm{H}$ synthase- 2 inhibition by nonsteroidal anti-inflammatory drugs. Mol. Pharmacol. 51:52-60.

53. Kurumbail, R.G., et al. 1996. Structural basis for selective inhibition of cyclooxygenase-2 by anti-inflammatory agents. Nature. 384:644-648.

54. Kalgutkar, A.S., et al. 1998. Aspirin-like molecules that covalently inactivate cyclooxygenase-2. Science. 280:1268-1272.

55. Serhan, C.N. 1997. Lipoxins and novel aspirin-triggered 15-epi-lipoxins (ATL): a jungle of cell-cell interactions or a therapeutic opportunity? Prostaglandins. 53:107-137.

56. Chiang, N., et al. 1998. Aspirin-triggered 15-epi-lipoxin $A_{4}$ (ATL) generation by human leukocytes and murine peritonitis exudates: development of a specific 15-epi-LXA 4 ELISA. J. Pharmacol. Exp. Ther. 287:779-790.

57. Marcus, A.J. 1999. Platelets: their role in hemostasis, thrombosis, and inflammation. In Inflammation: basic principles and clinical correlates. J.I. Gallin and R. Snyderman, editors. Lippincott Williams \& Wilkins. Philadelphia, Pennsylvania, USA. 77-95.

58. Clària, J., et al. 1998. Altered biosynthesis of leukotrienes and lipoxins and host defense disorders in patients with cirrhosis and ascites. Gastroenterology. 115:147-156.

59. Chavis, C., Vachier, I., Godard, P., Bousquet, J., and Chanez, P. 2000. Lipoxins and other arachidonate derived mediators in bronchial asthma. Thorax. 55(Suppl.):S38-S41.

60. Fiore, S., Maddox, J.F., Perez, H.D., and Serhan, C.N. 1994. Identification of a human cDNA encoding a functional high affinity lipoxin $\mathrm{A}_{4}$ receptor. J. Exp. Med. 180:253-260.

61. Gronert, K., Martinsson-Niskanen, T., Ravasi, S., Chiang, N., and Serhan, C.N. 2001. Selectivity of recombinant human leukotriene $\mathrm{D}_{4}$, leukotriene $\mathrm{B}_{4}$, and lipoxin $\mathrm{A}_{4}$ receptors with aspirin-triggered 15-epi-LXA $\mathrm{L}_{4}$ and regulation of vascular and inflammatory responses. Am. J. Pathol. 158:3-9.

62. De Caterina, R., Endres, S., Kristensen, S.D., and Schmidt, E.B. 1993. n3 fatty acids and vascular disease. Springer-Verlag. London, United Kingdom. 1-166.

63. Simopoulos, A.P., Leaf, A., and Salem, N., Jr. 1999. Workshop on the essentiality of an recommended dietary intakes for omega- 6 and omega3 fatty acids. J. Am. Coll. Nutr. 18:487-489.

64. 1999. Dietary supplementation with n-3 polyunsaturated fatty acids and vitamin $\mathrm{E}$ after myocardial infarction: results of the GISSI-Prevenzione trial. Gruppo Italiano per lo Studio della Sopravvivenza nell'Infarto miocardico. Lancet. 354:447-455.

65. Hill, D.J., Griffiths, D.H., and Rowley, A.F. 1999. Trout thrombocytes contain 12- but not 5-lipoxygenase activity. Biochim. Biophys. Acta. 1437:63-70.

66. Billman, G.E., Kang, J.X., and Leaf, A. 1999. Prevention of sudden cardiac death by dietary pure $\omega-3$ polyunsaturated fatty acids in dogs. Circulation. 99:2452-2457.

67. Clish, C.B., et al. 1999. Local and systemic delivery of a stable aspirintriggered lipoxin prevents neutrophil recruitment in vivo. Proc. Natl. Acad. Sci. USA. 96:8247-8252.

68. Corey, E.J., Shih, C., and Cashman, J.R. 1983. Docosahexaenoic acid is a strong inhibitor of prostaglandin but not leukotriene biosynthesis. Proc. Natl. Acad. Sci. USA. 80:3581-3584.

69. Hawkey, C.J. 1999. COX-2 inhibitors. Lancet. 353:307-314

70. Godson, C., et al. 2000. Cutting edge: lipoxins rapidly stimulate nonphlogistic phagocytosis of apoptotic neutrophils by monocyte-derived macrophages. J. Immunol. 164:1663-1667.

71. Sodin-Semrl, S., Taddeo, B., Tseng, D., Varga, J., and Fiore, S. 2000. Lipoxin $\mathrm{A}_{4}$ inhibits IL-1 beta-induced IL-6, IL-8, and matrix metalloproteinase- 3 production in human synovial fibroblasts and enhances synthesis of tissue inhibitors of metalloproteinases. J. Immunol. 164:2660-2666. 